https://doi.org/10.15407/ujpe64.8.745

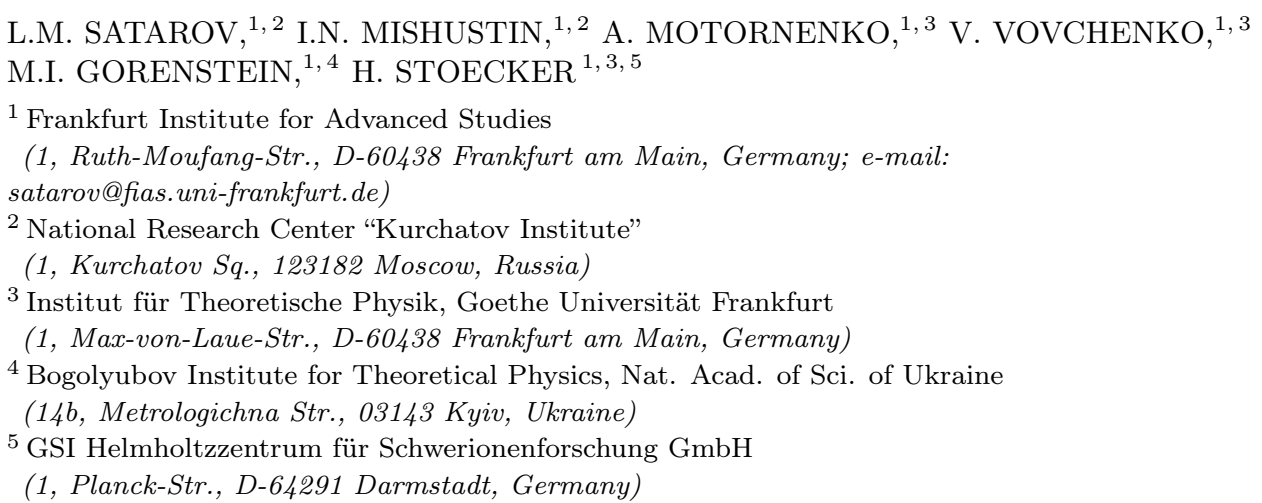

\title{
PHASE TRANSITIONS AND BOSE-EINSTEIN CONDENSATION IN ALPHA-NUCLEON MATTER
}

\begin{abstract}
The equation of state and the phase diagram of an isospin-symmetric chemically equilibrated mixture of $\alpha$ particles and nucleons $(N)$ are studied in the mean-field approximation. We use a Skyrme-like parametrization of mean-field potentials as functions of the partial densities of particles. The parameters of these potentials are chosen by fitting the known properties of pure $N$ - and pure $\alpha$-matters at zero temperature. The sensitivity of results to the choice of the $\alpha N$ attraction strength is investigated. The phase diagram of the $\alpha-N$ mixture is studied with a special attention paid to the liquid-gas phase transitions and the Bose-Einstein condensation of $\alpha$ particles. We have found two first-order phase transitions, stable and metastable, which differ significantly by the fractions of $\alpha$ 's. It is shown that the states with $\alpha$ condensate are metastable.
\end{abstract}

Ke y words: phase transitions, mean-field model, Bose-Einstein condensation, chemical equilibrium.

\section{Introduction}

At subsaturation densities and low temperatures, the nuclear matter has a tendency to the clusterization, when small and big nucleon clusters are formed under the conditions of thermal and chemical equilibrium. This state of excited nuclear matter is realized in nuclear reactions at intermediate energies known as the multifragmentation of nuclei $[1,2]$. It is believed that the clusterized nuclear matter is also formed in outer regions of neutron-stars and in supernova explosions [3].

In our recent paper [4], we studied the equation of state (EoS) of an idealized system composed entirely of $\alpha$-particles. Their interaction was described by a Skyrme-like mean-field potential. We have found

(C) L.M. SATAROV, I.N. MISHUSTIN,

A. MOTORNENKO, V. VOVCHENKO,

M.I. GORENSTEIN, H. STOECKER, 2019 that such a system exhibits two interesting phenomena, namely, the Bose-Einstein condensation (BEC) and the liquid-gas phase transition (LGPT). Earlier, the cold alpha matter was considered microscopically, by using phenomenological $\alpha \alpha$ potentials in Ref. [5].

However, by introducing such one-component system, one disregards a possible dissociation of alphas into lighter clusters and nucleons. The binary $\alpha-N$ matter in chemical equilibrium with respect to the reactions $\alpha \leftrightarrow 4 N$ was considered in [6], by using the virial approach. Due to the neglect of quantum statistics and three-body forces, such approach may be justified only at small baryon densities.

In this paper, we briefly discuss the results of our recent article [7], where we studied the isospin-symmetric $\alpha-N$ matter under the conditions of chemical equilibrium. The EoS of such matter was calculated in the mean-field approach, by using Skyrme- 
like mean-field potentials. In our study, we simultaneously take into account the LGPT and BEC effects.

\section{Mean-Field Model for Interacting $\alpha-N$ Matter}

Let us consider the iso-symmetric system (with equal numbers of protons and neutrons) composed of nucleons $(N)$ and alpha-particles $(\alpha)$. A small difference between the proton and neutron masses and the Coulomb interaction effects will be neglected. Our consideration will be restricted to small temperatures $T \lesssim 30 \mathrm{MeV}$. In this case, the production of pions and other mesons, as well as the excitation of baryonic resonances, become negligible. In addition, the masses $m_{N} \simeq 938.9 \mathrm{MeV}$ and $m_{\alpha} \simeq 3727.3 \mathrm{MeV}$ are much larger than the system temperature. Thus, a non-relativistic approximation can be used in the lowest order in $T / m_{N}$.

In the grand canonical ensemble, the pressure $p(T, \mu)$ is a function of the temperature $T$ and baryon chemical potential $\mu$. The latter is responsible for the conservation of the baryon charge. The chemical potentials of $N$ and $\alpha$ satisfy the relations

$\mu_{N}=\mu, \quad \mu_{\alpha}=4 \mu$,

which correspond to the condition of chemical equilibrium in the $N-\alpha$ mixture due to the reactions $\alpha \leftrightarrow$ $4 N$.

Let us denote, by $n_{N}$ and $n_{\alpha}$, the partial number densities of $N$ and $\alpha$, respectively. The baryonic density $n_{B}(T, \mu)=n_{N}+4 n_{\alpha}$, entropy density $s$, and energy density $\varepsilon$ can be calculated from $p(T, \mu)$, by using the equations

$n_{B}=\left(\frac{\partial p}{\partial \mu}\right)_{T}, \quad s=\left(\frac{\partial p}{\partial T}\right)_{\mu}, \quad \varepsilon=T s+\mu n_{B}-p$.

To characterize the relative abundances of $\alpha$ 's, we introduce their mass concentration $\chi=4 n_{\alpha} / n_{B}$.

In our mean-field model, we consider multiparticle interactions in the $\alpha-N$ matter, by introducing a temperature-independent "excess part" of the pressure $\Delta p$

$p=p_{N}^{\mathrm{id}}\left(T, n_{N}\right)+p_{\alpha}^{\mathrm{id}}\left(T, n_{\alpha}\right)+\Delta p\left(n_{N}, n_{\alpha}\right)$,

where the first and second terms on the right-hand side (RHS) are, respectively, the pressure of the ideal gas of nucleons and $\alpha$ 's. At known $\Delta p$, one can calculate the chemical potentials of $N$ and $\alpha$ as functions of $T, n_{N}, n_{\alpha}$. Solving further Eqs. (1), we get all thermodynamic quantities at given $T, \mu$.

Earlier, we suggested a similar scheme to describe the particle interactions in one-component $\alpha[4]$ and nucleon [8] matters. This corresponds, respectively, to the limiting cases $n_{N} \rightarrow 0$ and $n_{\alpha} \rightarrow 0$. In the case of binary $\alpha-N$ mixture, we use a generalized Skyrmelike parametrization [7] for the excess pressure

$\Delta p\left(n_{N}, n_{\alpha}\right)=-\left(a_{N} n_{N}^{2}+2 a_{N \alpha} n_{N} n_{\alpha}+a_{\alpha} n_{\alpha}^{2}\right)+$

$+b_{N}\left(n_{N}+\xi n_{\alpha}\right)^{\gamma+2}$.

Using Eqs. (3) and (4) and applying the thermodynamic relations, we get the expressions

$\mu_{N}=\widetilde{\mu}_{N}\left(T, n_{N}\right)-2\left(a_{N} n_{N}+a_{N \alpha} n_{\alpha}\right)+$

$+\frac{\gamma+2}{\gamma+1} b_{N}\left(n_{N}+\xi n_{\alpha}\right)^{\gamma+1}$

$\mu_{\alpha}=\widetilde{\mu}_{\alpha}\left(T, n_{\alpha}\right)-2\left(a_{N \alpha} n_{N}+a_{\alpha} n_{\alpha}\right)+$

$+\frac{\gamma+2}{\gamma+1} b_{N} \xi\left(n_{N}+\xi n_{\alpha}\right)^{\gamma+1}$.

Here, $\widetilde{\mu}_{i}\left(T, n_{i}\right)$ is the chemical potential of the ideal gas of $i$ th particles with the density $n_{i}(i=N, \alpha)$. The second and third terms on RHS correspond to the attractive and repulsive parts of mean-field potentials for $N$ ans $\alpha$. Note that, in the region of BEC, $\widetilde{\mu}_{\alpha}$ reaches its maximum possible value $\widetilde{\mu}_{\alpha}=m_{\alpha}$, and $n_{\alpha}$ contains the contribution of Bose-condensed $\alpha$ 's. In our calculations, we separate the states which are (meta)stable with respect to fluctuations of particle densities ${ }^{1}$.

To choose the model parameters $a_{N}, b_{N}, \gamma$, we fit the ground-state (GS) properties of the cold $(T=0)$ iso-symmetric nuclear matter. This is the state with zero pressure and minimal energy per baryon. We assume the GS-values $\mu_{N}=923 \mathrm{MeV}$, $n_{N}=0.15 \mathrm{fm}^{-3}[8]$ and choose $\gamma=1 / 6^{2}$. The parameters $a_{\alpha}, \xi$ are estimated, by using the properties of a cold $\alpha$ matter. We fit the values of density $\left(n_{\alpha}=0.036 \mathrm{fm}^{-3}\right)$ and binding energy per baryon $(E / B=-12 \mathrm{MeV})$ obtained in Ref. [5] for the GS of this matter.

The cross-term coefficient $a_{N \alpha}$ determines the attractive part of the $N \alpha$ mean-field potential. It is

1 For such states, the matrix $\left\|\partial \mu_{i} / \partial n_{j}\right\|$ is positive definite.

2 As shown in Refs. [7,8], such $\gamma$ gives reasonable values of nuclear compressibility.

ISSN 2071-0194. Ukr. J. Phys. 2019. Vol. 64, No. 8 

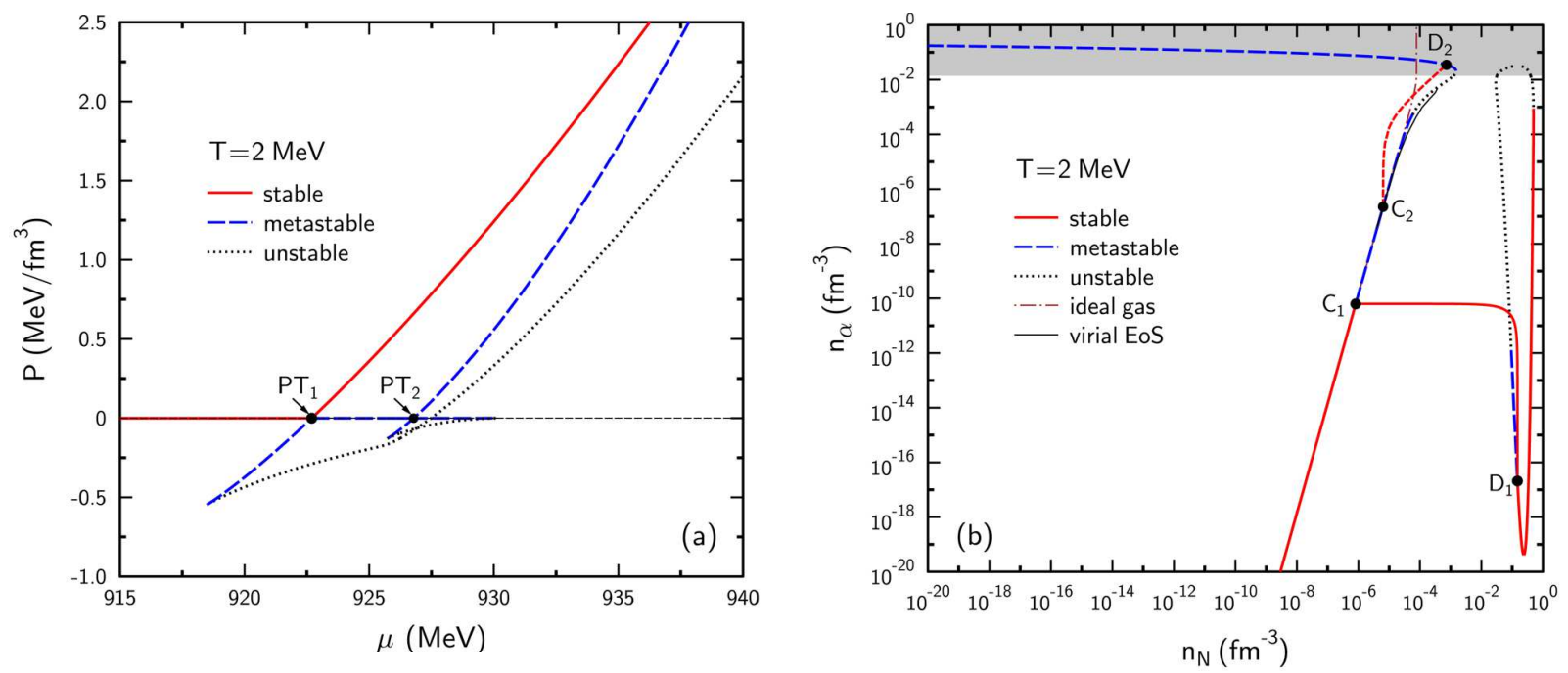

Fig. 1. Isotherm $T=2 \mathrm{MeV}$ of $\alpha-N$ matter on the $(\mu, p)(a)$ and $\left(n_{N}, n_{\alpha}\right)(b)$ planes. The stable, metastable, and unstable parts of the isotherm are shown, respectively, by the solid, dashed, and dotted lines. The dots $\mathrm{PT}_{1}$ and $\mathrm{PT}_{2}$ in $(a)$ show the positions of stable and metastable LGPT, respectively. The dash-dotted line in $(b)$ is calculated for the ideal $\alpha-N$ gas. Lines $C_{1} D_{1}$ and $C_{2} D_{2}$ correspond to the mixed-phase states of $\mathrm{PT}_{1}$ and $\mathrm{PT}_{2}$, respectively. The thin solid line represents the isotherm $T=2 \mathrm{MeV}$ from Ref. [6]

the only model parameter which is not fixed in our approach. To constrain this coefficient, we consider contours of the energy per baryon for the cold $\alpha-N$ matter on the $\left(n_{B}, \chi\right)$ plane. Our calculations show [7] that the properties of GS of such matter change drastically at some critical value $a_{N \alpha}=a_{*} \simeq$ $2.1 \mathrm{GeVfm}^{3}$. In the overcritical region $a_{N \alpha}>a_{*}$, the model predicts nonzero fractions of $\alpha$ in the GS of the $\alpha-N$ matter. In this case, the GS is stronger bound as compared to the pure nucleon matter. Apparently, this is in contradiction with phenomenological properties of the nuclear matter. Therefore, we consider only subcritical values of $a_{N \alpha}$. To probe the sensitivity to this coefficient, we made calculations for $a_{N \alpha}=1$ and $1.9 \mathrm{GeVfm}^{3}$. From the comparison with results of Ref. [6], we found that the latter value is more reasonable. Our "preferred" values of model parameters are given in Table 1.

\section{Phase Diagram of $\alpha-N$ Matter}

By substituting (5) and (6) into (1) and solving the resulting equations, we get the isotherms of the $\alpha-N$ matter for different $\mu$. At low enough temperatures, one obtains, in general, several solutions for the pressure at given $T, \mu$. Solutions with the largest (smallest) pressure correspond to stable (unstable) states. This is a typical situation for LGPT.
Figure 1, a represents the isotherm $T=2 \mathrm{MeV}$ on the $(\mu, p)$ plane. According to the Gibbs rule, the intersection points of (meta)stable branches of the pressure as functions of $\mu$ correspond to phase transitions (PTs). As one can see from Fig. 1, $a$, there are two PTs at $T=2 \mathrm{MeV}$. The first transition, $\mathrm{PT}_{1}$, occurs at a smaller baryon chemical potential than for $\mathrm{PT}_{2}$. The states on the dashed lines have smaller pressure as compared to states with the same $\mu$ on the solid lines. Therefore, the second transition $\mathrm{PT}_{2}$ is metastable.

Figure $1, b$ shows the same isotherm $T=2 \mathrm{MeV}$, but on the $\left(n_{N}, n_{\alpha}\right)$ plane. The shading represents the region of BEC. The states between $C_{1}$ and $D_{1}$ $\left(C_{2}\right.$ and $\left.D_{2}\right)$ are mixed-phase states for the stable (metastable) PT. As compared to $\mathrm{PT}_{1}$, the concentrations of $\alpha$ are much larger for the mixed-phase states of $\mathrm{PT}_{2}$. A strong suppression of $\alpha$ is predicted at large nucleon densities. According to our calculation, BEC states are metastable (see the dashed line in the shaded domain).

Table 1. Model parameters

\begin{tabular}{|c|c|c|c|c|c|}
\hline$\gamma$ & $\begin{array}{c}a_{N}, \\
\mathrm{GeV} \mathrm{fm}^{3}\end{array}$ & $\begin{array}{c}b_{N}, \\
\mathrm{GeV} \mathrm{fm}^{3.5}\end{array}$ & $\begin{array}{c}a_{\alpha}, \\
\mathrm{GeV} \mathrm{fm}^{3}\end{array}$ & $\xi$ & $\begin{array}{c}a_{N \alpha}, \\
\mathrm{GeV} \mathrm{fm}^{3}\end{array}$ \\
\hline $1 / 6$ & 1.17 & 1.48 & 3.83 & 2.006 & 1.9 \\
\hline
\end{tabular}



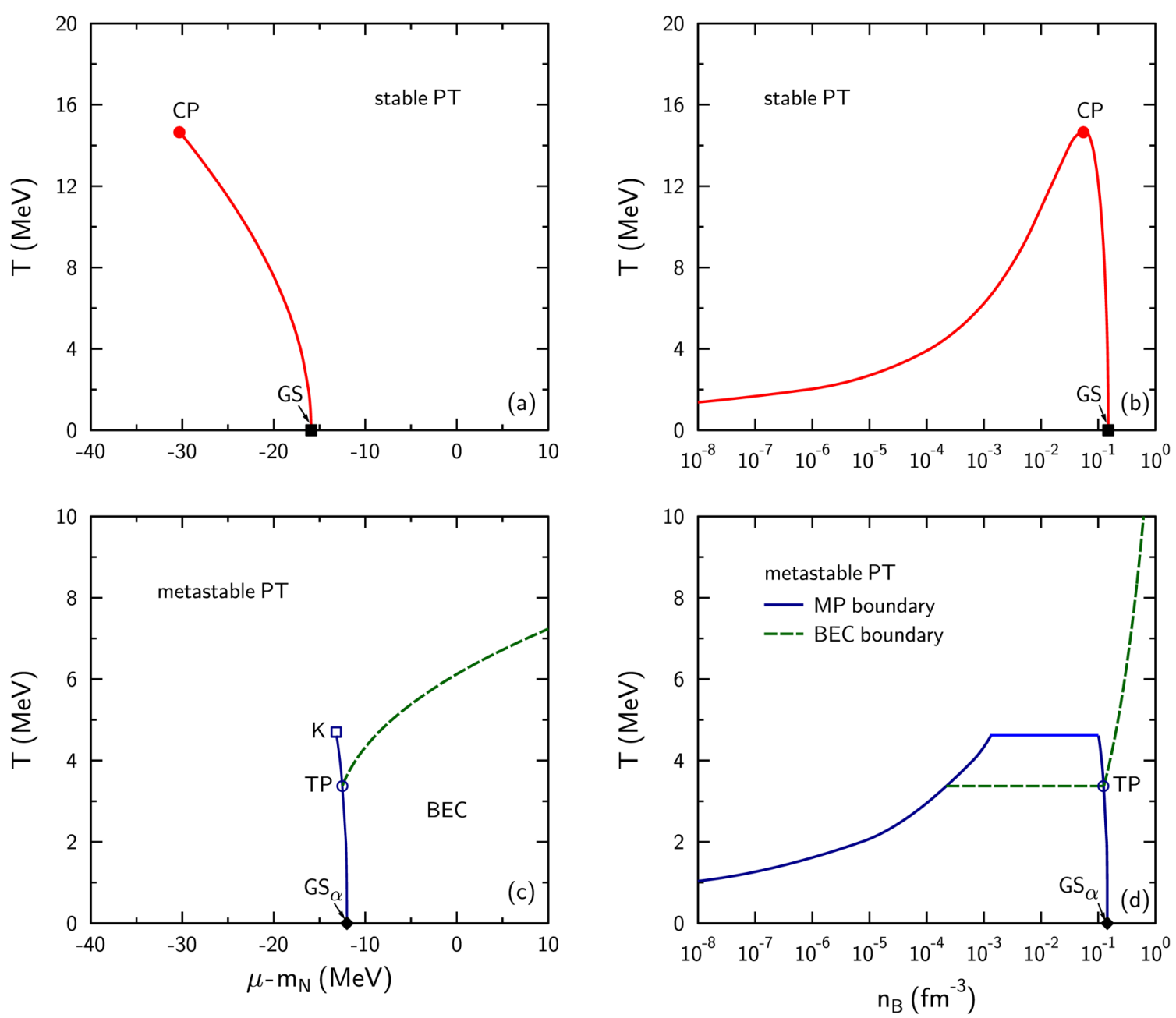

Fig. 2. Left panels: critical lines of stable $(a)$ and metastable $(c)$ PT of the $\alpha-N$ matter on the $(\mu, T)$ plane. Right panels: boundaries of the mixed phase for stable $(b)$ and metastable $(d)$ PT of the $\alpha-N$ mixture on the $\left(n_{B}, T\right)$ plane. Full circles in $(a)$ and $(b)$ show positions of the critical point. The dashed lines in $(c)$ and $(d)$ represent boundaries of the BEC region. The open square (circle) marks the end (triple) point of the metastable PT. The full squares and diamonds show, respectively, the GS positions for the pure nucleon and pure alpha matters, respectively

Table 2. Characteristics of phase transitions in $\alpha-N$ matter

\begin{tabular}{|c|c|c|c|c|c|c|c|}
\hline \multicolumn{3}{|c|}{ Stable PT } & \multicolumn{3}{c|}{ Metastable PT } \\
\hline $\begin{array}{c}T_{\mathrm{CP}}, \\
\mathrm{MeV}\end{array}$ & $\begin{array}{c}\mu_{\mathrm{CP}}, \\
\mathrm{MeV}\end{array}$ & $\begin{array}{c}n_{B \mathrm{CP}}, \\
\mathrm{fm}^{-3}\end{array}$ & $\chi_{\mathrm{CP}}$ & $\begin{array}{c}T_{K}, \\
\mathrm{MeV}\end{array}$ & $\begin{array}{c}\mu_{K}, \\
\mathrm{MeV}\end{array}$ & $\chi_{K}$ & $\begin{array}{c}T_{\mathrm{TP}} \\
\mathrm{MeV}\end{array}$ \\
\hline 14.7 & 908.6 & $5.3 \times 10^{-2}$ & $6.9 \times 10^{-2}$ & 4.6 & 925.7 & $0.46-0.86$ & 3.4 \\
\hline
\end{tabular}

Analyzing the results at different $T$, we get the phase diagram of the $\alpha-N$ matter. The stable and metastable parts of this diagram are shown in the upper and low panels of Fig. 2. Characteristics of $\mathrm{PT}_{1}$ and $\mathrm{PT}_{2}$ are shown in Table 2 . Note that the metastable $\mathrm{PT}$ disappears at the temperature $T_{K} \simeq$ $\simeq 5 \mathrm{MeV}$ which is much less than the critical temperature $T_{\mathrm{CP}} \simeq 15 \mathrm{MeV}$ of the stable PT.

\section{Conclusions}

Our model describes both the phase transitions and BEC of the $\alpha-N$ matter. The results of this paper may be used for studying the nuclear cluster production in heavy-ion reactions, as well as in astro-

ISSN 2071-0194. Ukr. J. Phys. 2019. Vol. 64, No. 8 
physics. We think that the present formalism can be also used for the binary mixtures of fermionic atoms and bosonic molecules, like $\mathrm{H}+\mathrm{H}_{2}$ or $\mathrm{D}+\mathrm{D}_{2}$.

1. J.P. Bondorf. Heavy ion reactions between 30 and a few hundred MeV/nucleon. J. Phys. Colloq. 37, 5 (1976).

2. J.P. Bondorf, A.S. Botvina, A.S. Illjinov, I.N. Mishustin, K. Sneppen. Statistical multifragmentation of nuclei. Phys. Rep. 257, 133 (1995).

3. J.M. Lattimer, F.D. Swesty. A generalized equation of state for hot, dense matter. Nucl. Phys. A 535, 331 (1991).

4. L.M Satarov, M.I. Gorenstein, A. Motornenko, V. Vovchenko, I.N. Mishustin, H. Stoecker. Bose-Einstein condensation and liquid-gas phase transition in strongly-interacting matter composed of $\alpha$ particles. J. Phys. G 44, 125102 (2017).

5. J.W. Clark, T.-P. Wang. Theory of $\alpha$ matter. Ann. Phys. 40, 127 (1966).

6. C.J. Horowitz, A. Schwenk. Cluster formation and the virial equation of state of low-density nuclear matter. Nucl. Phys. A 776, 55 (2006).

7. L.M. Satarov, I.N. Mishustin, A. Motornenko, V. Vovchenko, M.I. Gorenstein, H. Stoecker. Phase transitions and Bose-Einstein condensation in alpha-nucleon matter. Phys. Rev. C 99, 024909 (2019).
8. L.M. Satarov, M.N. Dmitriev, I.N. Mishustin. Equation of state of hadron resonance gas and the phase diagram of strongly interacting matter. Phys. At. Nucl. 72, 1390 (2009).

Received 08.07.19

Л.М. Сатаров, І.Н. Мішустін, А. Моторненко,

В. Вовченко, М.I. Горенштейн, Х. Штокер

ФАЗОВІ ПЕРЕТВОРЕННЯ

ТА КОНДЕНСАЦЯ БОЗЕ-ЕЙНШТЕЙНА

В АЛЬФА-НУКЛОННІЙ МАТЕРІЇ

$\mathrm{P}$ е $з$ ю м е

Рівняння стану та фазова діаграма ізоспін-симетричної хімічно рівноважної суміші $\alpha$ частинок та нуклонів $(N)$ вивчається в наближенні середнього поля. Ми застосовуємо параметризацію Скірма для потенціалів середнього поля як функцій парціальних густин частинок. Параметри цих потенціалів знайдені як результат підгонки відомих властивостей чистої $N$ - та чистої $\alpha$-матерії при нульовій температурі. Вивчена чутливість результатів до вибору величини $\alpha N$ притягання. Фазова діаграма $\alpha-N$ суміші вивчається з особливою увагою до процесів фазового перетворення рідина-газ та конденсації Бозе-Ейнштейна для $\alpha$-частинок. Ми знаходимо два фазові перетворення, стабільний та метастабільний, які значно відрізняються концентраціями $\alpha$-частинок. Показано, що стани $3 \alpha$ конденсатом є метастабільними. 